\title{
Spoken Grammar: Meaning, Principles and Characteristics
}

\author{
${ }^{1}$ Ru'a Salim Mahmood, ${ }^{2}$ Hussein Ali Ahmed Allo \\ ${ }^{1}$ Ninevah General Directorate of Education \\ 2Department of English, College of Languages/ Nawroz University, Duhok, Kurdistan Region, Iraq
}

\begin{abstract}
The terms Spoken Grammar was coined by the two corpus grammarians, Ronald Carter and Mike McCarthy. In the 19th century, it came under the impact of a number of local dialects represented by the cockney dialect in London, and the Lothian dialect in Edinburgh. The discussions, debates and studies on Spoken grammar have led to the specification of three main viewpoints concerning the existence of this types of grammar. The viewpoints entail that (1) grammatical rules do not govern spoken language, which is disorderly and disordered; (2) Speaking English lacks a distinct grammar. It has the same syntax as written English grammar; and (3) spoken language is regulated by a separate grammar with its own set of rules and conventions; i.e., it has its own grammar represented by its own set of rules, regulations, and classifications compared to those of the written language. $\mathrm{T}$ validate or refute the implications of the preceding viewpoints; relevant literature concludes that spoken grammar is quite prevalent in everyday conversational spoken English. It is characterized by being more flexible and less strict compared to written grammar. This is so because the informal context of using spoken grammar makes it have a syntax that varies from the traditional written grammar in a number of aspects. This purely theoretical research aims at shedding light on the definition, meaning, principles and the main characteristics of spoken grammar. The emphasis on the distinctive features of spoken grammar has triggered the researchers to focus on a further point of discussion, namely the differences between spoken and written grammar. To substantiate such differences, examples from closely relevant grammatical literature have also been provided. The research ends with some concluding points drawn upon from the preceding discussed and presented points.
\end{abstract}

Keywords: Spoken Grammar, Written Grammar, Conversational spoken English, Grammatical rules.

\section{Introduction}

Spoken grammar (henceforth SG) is a thorny linguistic domain. It does not vary from one cultural context to the next, but rather individual- and emotion-oriented, since it has higher ego involvement than written grammar. It is rooted in the speakers common context and depends on their interpersonal relationships. Added to that, SG is unplanned and accidental, interactive, and ambiguous and avoids elaboration. Such features of SG has made it quite uneasy to base many features of SG on useful and digestible development rules (Leech, 2000, Timmis, 2005; Goh, 2009; Leech, 2009).

As a matter of fact, SG, in terms of its word order, more flexible and is much less strict than written grammar. This is so because informal and conversational spoken English has a syntax that, in a variety of respects, varies from the traditional written grammar. Examples of such variations are fronting when the object, the complement or an adverbial are placed at the beginning of the sentence or clause and/or tailing when the already mentioned elements are put at the end of the sentence or clause. called tailing. In both cases, the subject and/or the emphasis are transferred.

SG has been differently viewed as it is claimed that (1) There is lacking of grammar in the SG; (2) Spoken English has a grammar that is limited to it; and (3) there is a set of activities are tailored for the teaching of SG.

This research aims at shedding light on SG which has been of noticeable prevalence in conversational spoken English. It further aims at investigating the nature of this grammar in terms of its main features. Another aim of this study is highlighting the differences between SG and written grammar. The teaching of SG is a further topic that is intended to be 
covered in the current research.

This research is limited to the study of SG in terms of its definition, nature, features and way of teaching. Any topics that are not of relevance to the aforementioned topics lie beyond the scope of the current research.

The current research is expected to be of value to teachers and students of EFL at university level. They may find in the information presented a source and a means of better understanding of the topic SG and the aspects of difference between this grammar and written grammar.

\section{Spoken Grammar}

\subsection{Historical Background}

Ronald Carter and Mike McCarthy, two corpus grammarians, were the first to coin the word "spoken grammar." According to Sweet (1890), in the 19th century, SG came under the influence of a set of local dialects represented, in the main, by the cockney dialect in London, and the Lothian dialect in Edinburgh.

SG witnessed change from generation to generation, and even among speakers of the same generation residing in the same place and having the same social status.

Introducing a grammar based on spoken language has recently been a noticeable trend in research on corpus linguistics, whereby linguists can easily see what takes place in speech as corpus-resources and computerized databases that subsume all the available facets of speech. Both spoken and written English examples from "true life" "The Longman Grammar of Spoken and Written English," published in 1999, is an example of a current reference book on English grammar that focuses on an extensive corpus.

Furthermore, steady advances in corpus linguistics and discourse analysis research have identified the existence of SG, specified its characteristics, and highlighted the differences between standard written grammar and the SG used by native speakers in conversation. As a result of these advancements, conflicting views on the nature of SG have been identified; a point that will be tackled in the following pages.

\subsection{Definition}

The expression 'spoken grammar' (SG) was first coined by both Corpus grammarians Ronald Carter and Mike McCarthy. As for the definition of SG, Goh (2009: 23) defines SG as a distinct grammar with characteristics that vary from those of regular written grammars. For Paterson (2019), SG refers to the elements of conversation grammar that have been specified by the people concerned, namely teachers and corpus researchers, yet such elements are still waiting to be listed as a part of the regular syllabuses of teaching.

\subsection{Importance}

Grammar is a collection of structural rules that influence the composition of sentences, words, and terms in any given language. It is the systematic study and description of a language that enables us to comprehend how words combine to form sentences with their constituent parts.

By bringing to the forefront the already features of SG, learners are exposed to real language, not that in course book English. This is particularly important on the courses where reading forms the main channel for learners' getting of input.

Some people think that correct English grammar matters only to teachers and is of no real importance in daily life. This is certainly not true and has been rejected by Cheshire (1999) who argues that grammar is the foundation for communication since message relayed with the correct grammar is easier to understand in terms of its purpose and meaning. As such, knowledge of the grammar of the language is necessary for communication, i.e. expression of one's self. This is added to the fact that writing that is poorly punctuated and contains grammatical errors is difficult to read and comprehend. If a reader needs to 
go back and reread a sentence many times because they do not understand what it means, their reading experience is destroyed, and they are more likely to misinterpret the argument or even give up and avoid reading.

Finally, tackling everyday language not that in the conventional teaching materials is supposedly more relevant to students' lives as it is the language they hear in the movies and songs, and use when chatting on social media.

SG may help LLs convey more sophisticated ideas at an earlier skill level by lessening the intensity of cognitive demand in an area that is already quite difficult. Furthermore, in the language classroom, introducing NS models of spoken grammar should help alleviate the affective pressure on LLs to make perfect sentences every time they speak (Goh, 2009).

\section{Perspectives on the Existence of Spoken Grammar}

According to Biber et al. (1999), there are three different perspectives on the existence of spoken grammar:

According to the first viewpoint, grammatical rules do not govern spoken language, which is disorderly and disordered. To put it another way, spoken English lacks any grammatical structure; it is grammatically inchoate. This perspective is based on the traditional view of associating grammar with written language, and it is followed by examples such as the following, which comes from the Longman spoken corpus: Geoffrey Leech's essay, English Grammar in Conversation:

i. Do you know erm you know where the erm go over to er go over erm where the fire station is not the one that white white.

Speaking English, according to the second point of view, lacks a distinct grammar. It has the same syntax as written English grammar. Although the words used in speech and writing differ (for example, more formal written registration, more phrasal verbs in speech, etc.), they are all bound by the same grammar rules.
On this basis, it is viewed that conversation, like written language, employs entities such as prepositions, modals, noun phrases, and relative clauses. So, if you assume, as many do, that the use of grammar is part of the variations in frequency rather than the grammatical structure itself, it is natural to think of English grammar as a single system, whose use of various types of writing in conversational effects can be contrasted. In other words, SG is regarded as a very particular application of English's standard grammar.

The third point of view has got a lot of coverage in recent years. This perspective argues that spoken language is regulated by a separate grammar with its own set of rules and conventions. In other words, spoken English has its own grammar represented by its own set of rules, regulations, and classifications compared to those of the written language.

Finally, Carter and McCarthy (1995) argue for a profoundly different approach to grammar than that which has become familiar as a result of the conventional focus on written language in the treatment of spoken language. They suggest a linear model that travels through time dynamically, rather than the more traditional architectural model of unit hierarchies. On this basis, they focus on grammatical features of spoken language that they feel have been ignored by standard grammars rooted in the 'written tradition.' They further argue that structures inherent in speech were mistakenly handled until the introduction of the spoken computer corpus; a point tha had led to their absence from written grammar used by English learners worldwide. Consider the following structures:

ii. the 'dislocated topic' of This little shop ... it's lovely..

or

iii. the 'wagging tail' of Oh I reckon they're lovely. I really do whippets.

The smooth progression of speech, with just a narrow 
space for the speaker to plan what to say, and often triggering retrospective add-ons, helps in justifying the presence of such structures. Accordingly, Carter and McCarthy (1995) suggested a structural model for the clause that includes a pre-clause subject and a postclause tail, as well as a pre-clause subject and a postclause tail in addition to the core clause itself.

\section{Spoken Grammar Vs. Written Standard Grammar}

Investigating such a topic requires, in the first place, posing the following set of questions:

$i v$. Is there a difference between spoken and written grammar?

v. Are the differences significant and important to EFL teachers?

vi. What are teachers' choices for coping with the existence of such differences?

Standard English has been a focal point of attention for linguists who, in addition to their involvement in written English, "the fact that standard English is mostly a written variety does not bode well for their comprehension of structures that are more akin to spoken English than written English" (Cheshire, 1999). In the evolution of English, the relationship between spoken and written English has nearly come full circle. In the Middle Ages, written English was mainly used as a means to (1) represent earlier spoken words or (2) set permanent records of events, thoughts, or spoken exchanges. The $17^{\text {th }}$ century witnessed the development of the independent identity of the written word; a phenomenon that went on with more advancement in the $18^{\text {th }}, 19^{\text {th }}$ and the first half of the $20^{\text {th }}$ centuries. Just parallel to that, and until at least the end of the 19 $9^{\text {th }}$ century, individuals with social and educational ambitions heavily heeded the spoken rhetorical skills. Since World War II, everyday speech, at least in the USA, was gradually reflected by written English. Though computers were not used to initiate this trend, the later was accelerated by writing on-line with computers. For the time being, contemporary spoken and written English are losing their identities as distinct forms due to the fact that writing increasingly mirrors informal speaking of language (Baron, 2000).

Likewise, the variation in the word order of SG makes it less effortful as compared with written grammar; this is good news for language learners who are after the development of their speaking skills. For instance, in written grammar, prepositions, pronouns, and articles for the 100 most common words in written grammar. They are the words that characterize the correct grammatical structure of sentences, while spoken English is characterized by significant portion of the top 100 words, namely verbs (The Cambridge International Corpus (CIC). It is worthy to note that this corpus comprises 1 billion words, passages taken from books, journals, and magazines, spoken - casual, business, and academic exchanges and data from Cambridge TESOL exams.

The argumentative distinction between SG and written grammar is attended to in terms of that between formal and informal grammar. It is worthwhile that such arguments have labeled SG as inchoate and that is almost the same as written grammar since the use of grammar rather than a particular framework is forms the focal point of emphasis. Additionally, the distinction is theoretically important as far as language use is concerned since the written grammar use in speech and SG use in writing can be contextually incorrect and lead to confusion. Simply put, people do not usually speak the way they write, and they do not write the way they speak.

Hird (2016) states that much of the grammar we teach is centered on subject-verb-object word order in terms of basic syntax. While the grammar of more official English tends to follow this traditional word sequence, the grammar of more casual and conversational spoken English can be quite different in a number of ways. We can move the focus and emphasis of an utterance by placing the object, complement, or adverbial at the beginning of the phrase or clause 
(fronting) or at the conclusion of the phrase or clause (tailing). In its most basic form, fronting is when we insert something that normally comes at or near the end of a clause (e.g., the object, complement, adverbial, or question-word phrase) in front of it. This is done to draw attention to the item, complement, and so on. It can also improve group cohesion. Here are some more examples of simple fronting, some of them from written online conversations.

vii. Fifty pounds that cost me!

viii. This one I've had for ages.

ix. What it's based on, I don't know.

Tailing (or utilizing a tail) occurs when the subject comes after the main phrase and is introduced with a pronoun, as in He...this man in the example below. After the clause, restating the complete subject draws attention to it and underlines, amplifies, or clarifies it. Here are some more examples.

$x$. It's always pretty good, the food here.

xi. He's a great drummer, Brian Downey.

Another common pattern is It ... that or this.

xii. It's a great film, that.

xiii. It was fun, that.

xiv. It's a nice place, this.

Paraphrasing is a further feature that distinguishes SG from written standard grammar. Hird (2016) maintains that learners of English are very likely to encounter this syntactic component of SG outside of the classroom. And it appears to be a feature that some people are quick to notice and use. So, whether we teach this part of spoken language explicitly or simply deal with it as it arises, it is helpful to have some short explanations on hand, as well as a few easy examples and activities to assist show, explain, and practice the language. Consider the following examples:

\section{xv. (He) Always reminds me of Alex, that guy. \\ xvi. (It) Takes me right back, that album.}

To conclude, the key explanation for SG is that it varies from written grammar in terms of approach. Carter and McCarthy (2006) state that familiarity with the use of written grammar entails the existence of a case for a linear grammar model with separate and distinct spoken grammar. Yet over time, the model has developed into a dynamic type of grammar structure that is easily adaptable and flexible for language use.

\section{Characteristics of Spoken Grammar}

According to Crystal (2003), conversational grammar (spoken grammar) is generally thought of as "incorrect," "inferior," or at best, "less significant" than standard grammar. Traditional grammar's legacies have contributed to such a gloomy outlook. Since spoken language does not "conform to the laws" of written language, it is full of "imperfections" and has "less" grammar, according to the latter.

In fact, there are many substantial differences between speaking and writing, but these distinctions do not mean that conversational grammar (spoken grammar) is less accurate than writing and formal speaking grammar (standard grammar). Rather, such distinctions mean that each form of grammar has its own distinct characteristics and features that set it apart from the others. To put it another way, while traditional grammar meets the needs of writing and formal speaking processes that are typically prepared ahead of time, spoken grammar is well suited to the conversational process, which happens in real time with very little time for thorough preparation. As a consequence, each form of grammar is a required system for representing and promoting the expression of meanings for its particular type of language.

Based on the preceding aspects of the differences between spoken grammar and standard written grammar, Paterson (2019) claims SG has a number of features that differentiate it from standard written grammar:

- SG includes contractions that are not explicitly permissible in written grammar, such as "I'll," "don't," and "can't."

- Slang terms and colloquialisms are also used in spoken grammar. 
- Prepositions are used against the strict written grammar rules. An example is prepositions use at the beginning of sentences.

- The absence of a grammatical syntax such as the perfect past tense which is almost exclusively present in written grammar.

- The spontaneous handling of SG as a part of immediate language production makes it have several grammatical errors that are unacceptable in written grammar which is characterized by its precision, less margins for error, more advanced vocabulary and presentation style.

- Communication of concepts at a lower level of interaction makes SG more open and duly more communicative.

- Furthermore, the Ellipsis feature exists in spoken grammar, which is the absence of one or more words in a clause.

xvii. Any luck? Instead of "Did you have any luck?"

- Repetition is more common in SG compared to written grammar. This is so since there is less or no time to plan for what will be said in real time because spoken language is spontaneously generated. An example is

xviii. It is a funny place, this town.

- Vagueness does not exist in SG compared to written grammar where vague language is more likely to be the sign of a skilled and sensitive language producer than a lazy one (Carter and McCarthy, 2006: 202).

- Absorption of the historical present tense by SG. A more dramatic and vivid effect is created by the use of present tenses to narrate past events: (19) I get to my car and then I realise that I've left my car keys at home so I go back home and guess what?

- SG uses of discourse markers that are different from those used in written English:

xix. Well..., anyway...I mean... You know

NB. "You know" is the most frequent word combination in English

- SG uses formulaic language as exchanges in conversation are often made up of formulaic phrases rather than full sentences.

$x x$. That's fine. What time? What about...?

- There is shared understanding in spoken language; that is, determining what the other person knows and expressing a common point of view.

- SG requires constant checking that is not needed in writing. Without constant checking, i.e. verification, speech will end up in monologue instead of dialogue. Examples of checking phrases are:

xxi. 'Do you see?' or 'You know what I mean'.

- Response tokens such as Good, definitely, fantastic, unquestionably, which are necessary for successful oral communication, are seldom used in in SG compared to written grammar.

- Answer Questions are prevalent in SG in a way that they do not ask for answers, but rather share feelings or personal attitudes in response to what has been said.

- In spoken English, verbs form many of the top 100 words are verbs. For instance, the verb "know" is the $14^{\text {th }}$ most commonly used word in spoken British English and the 22nd in American English. "know", through "you know", mostly lacks justification for its use in speech. The verbs "see' and "mean" are further examples in this respect. Contrariwise, prepositions, pronouns, and articles are the 100 most common words in written grammar that give sentences the proper grammatical structure.

- SG makes use of a number of noun phrases and multi-topic constructions that sound perfect when spoken aloud, i.e. individuals have no difficulty to understand them in conversation, yet they seem odd when written down. Consider the following example: 
xxii. His cousin in London, her boyfriend, his parents, bought him a car for his birthday.

- In SG, there is common use of vocative forms by speakers to refer specifically to someone or something. Consider the examples below where the listener(s) in the conversational phase use their names, adjectives, or pronouns that specifically address them.

xxiii. [Mike is calling Steve to help him fix the remote control.]

Mike: Steve, come and see this!

Steve: What is it, Mike?

xxiv. [A Father allowing his daughter to attend a party]

Father: You can go, honey, but don't be late.

Daughter: OK, Dad.

- SG uses statements as questions. As such, there are sentences that sound like questions but with no subject-verb reversion. It is believed that such structures are more effective at conveying knowledge than conventional questions. Paterson (2019) provides the following examples:

$x x v$. [Friends talking about a past incident]

A: I rang the police last night.

B: You did what?

xxvi. [Business partners discussing a problem]

A: I talked to them, and they promised to help us.

B: Are you kidding?

A: It was the only way.

\section{Teaching Spoken Grammar}

Emerson (1896) asserted the inadequate teaching of English since the bulk of instruction is based on written grammar through the regular focus on written examples and negligence of the knowledge and trust to use spoken grammar. He further states that learners should be provided with choices between written and SG since the latter, i.e. SG due to the significance of its interpersonal implications and the suitability of the methodologically inductive learning compared to the 3Ps approaches adopted in conventional grammar books, namely Presentation-Practice-Production.

In a study cited in TESOL (2003), it was found out that both teachers and learners value the teaching of all aspects of grammar since learners must be enabled, on the basis of their individual educational needs, to communicate in an appropriate and efficient manner as much as possible. In other words, it is important to use SG in English teaching on the basis of the proposed educational outcomes and teaching goals and to achieve competent language competence. Such an inclusion procedure may also inspire encouragement and influence learners' outcomes if they believe like they are fully participating in the language in a manner that is observable.

Leech (2000) argues that SG is but functional grammar and that it has been created by native speakers to minimize the cognitive demand on the speaker. He further argues that the combination of shared context and interactive characteristics on one hand, and the linguistic element of conversation on the other hand, enable speakers to make use of use many grammatical shortcuts the use of which is not allowed in a decontextualized, written grammar. Added to that, according to Goh (2009: 307), while learners excessive emphasis on language form may hinder communication, their familiarity with certain linguistics phenomena such as ellipsis or flexible positioning in SG may aid in overcoming the psychological barrir and speak with no overthinking things.

Teachers' proper teaching and explanation of the differences between written grammar and SG may be a further source of help in both teaching SG and enabling learners to overcome anxiety while speaking. Yet, if teachers, as Mumford (2009: 139) outlines, "insist on students conforming to 'written grammar' norms while speaking, we may be making their task more difficult again"). 
On his part, Zhou (2006) presents a teaching model based on what he labels as the "Production Approach" that aims at improving language learners' general oral production skills. Here, language learners' analysis of native speakers' examples, imitation of such examples and comparison of their own, i.e language leaners' recorded presentations to the NS models, can assist in a better interaction between the learners and the native speakers as learners would find themselves in contexts where more realistic and varied language is used.

According to Mumford (2009), the teaching or nonteaching of SG can be viewed in terms of the following approaches:

The 'Lingua Franca Approach' recommends no teaching of SG. It is based on many educators' position against the teaching of SG and sets out of the assumption that native speakers' models of SG are are inadequate for classroom FL teaching and that individual cultural norms are irrelevant for language teaching in an international environment (Mumford, 2009; Usó-Juan and Martínez-Flor, 2008). Such a stand is also aided by the belief that the teachers who are adopting the 'Lingua Franca' approach' teach the FL in contexts characterized by a minimum use of native speakers' interaction.

The 'Passive Approach' emphasizes the introduction and application of native speakers models of SG rather than their production in language training. On this basis, those who follow this approach, Timmis (2005) is an example, highlight the importance of exposure to SG listening activities, noticing activities, and language discussion activities; The same point is emphasized by Carter and McCarthy (1994: 25) who recommend the inclusion of noticing tasks and listening comprehension activities, as well as requests to "expose learners to natural spoken data wherever possible and help them become observers of the grammar". The same point is advocated by Brown and Yule (1983) who encourage the early production of SG as it has a limited syntax and a broad vocabulary.

Since it is essential for learners to realize the importance of such an inclusion of SG in English language teaching, the adoption of some wellbalanced and calculated procedures can be effective and fruitful:

- Integrating language elements of spoken English in EFL teachers' education.

- Developing learners' understanding of the differences between the two grammars in their own language,

- Using corpora to identify and teach the most appropriate words and phrases.

- Paying attention to examples.

- Making mental notes of how the expressions are used and looking for further examples.

- Making use of free practice.

- Using controlled practice by learners' giving of appropriate answers to things they hear, such as a piece of good news or a piece of bad news.

To be more specific, the following exercises can be used to teach SG in the classroom:

- Organizing formulaic phrases to create short dialogues.

- Investigating a variety of advantageous applications (e.g. to end a conversation, to introduce a story, etc.).

- Using phrases like kind of, or something similar to make a dialogue sound more hazy.

- Using only things, objects, and bits and as many adjectives and verbs as the teacher can.

- Mentioning all examples of the language spoken when watching a movie scene (TESOL, 2003).

To conclude, and in line with Emerson (1896), the teaching of English grammar should not be limited to that of the nature of language and the history of English._The spoken form of the language should also be accounted for as it is not right for the educated mind to attend to the written form of the language and forget about its spoken form. The latter should play a role in 
developing learners' understanding and tackling of the varied language aspects.

\section{Conclusion}

The phrase "spoken grammar" was first coined by the corpus grammarians Ronald Carter and Mike McCarthy. SG subsume elements of English language at large and its grammar in particular that teachers and corpus researchers are already aware of, yet have not been set as components of the teaching curriculum. There are three different viewpoints on the essence of SG: spoken language is devoid of grammar; SG is close to written grammar; and spoken English has its own grammar. The controversy about formal vs. informal grammar is at the core of the distinction between SG and written grammar, especially in terms of SG having a range of distinctive features that sets it apart from conventional written grammar. Finally, the adoption of a number of approaches and methods can facilitate the teaching of SG and make it play a role in developing learners' understanding of the varied aspects of English language.

\section{References}

1. Baron, N. S. (2000). Alphabet to Email: How Written English Evolved and Where It's Heading. New York: Routledge.

2. Biber, D., Johansson, S., Leech G., Conrad, S., and Finegan, E. (1999). Longman Grammar of Spoken and Written English. London: Longman.

3. Brown, G. and Yule, G. (1983). Discourse Analysis. Cambridge: Cambridge University Press.

4. Carter, R. and McCarthy, M. (1995). Grammar and the spoken language. Applied Linguistics, 16, 141-158.

5. Carter, R. and Mcarthy, M. (2006). Cambridge Grammar of English: A Comprehensive Guide. (1 $1^{\text {st }}$ ed.). Cambridge: CUP.

6. Cheshire, J. (1999). "Spoken Standard English", Standard English: The Widening Debate, ed. by Tony Bex and Richard J. Watts. New York: Routledge,

7. Crystal, D. (2003). The Cambridge Encyclopedia of the English Language, (2nd ed.). Cambridge: Cambridge University Press.

8. Emerson, O. F. (1896). The Teaching of English Grammar.

9. Goh, C. (2009). "Perspectives on spoken grammar". ELT Journal 63 (4): 303-312.

10. Hird, J. ( 2016). It's different, spoken grammar. Oxford: Oxford University Press ELT

11. Paterson, K. (2019). "9 thoughts on "It's different, spoken grammar", Grammar Fiction. Oxford: Oxford University Press.

12. Leech, G. (2000). Grammars of spoken English: New outcomes of corpus-oriented research. Language Learning, 50 (4): 675-724.

13. Mumford, S. (2009). An analysis of spoken grammar: The case for production. ELT Journal 63 (2): 137-144.

14. TESOL (2003). Designing Natural Spoken English Courses. Spain: Valencia..

15. The Cambridge International Corpus $\left.(\mathrm{CIC})^{*}\right)$.

16. Timmis, I. (2005). Towards a framework for teaching spoken grammar. ELT Journal 59 (2): 117-125.

17. Usó-Juan, E. and Martínez-Flor, A. (2008). Teaching learners to appropriately mitigate requests. ELT Journal, 62(4), 349-357.

18. Zhou, Q. (2006). Application of a discourse approach to speaking in teaching of conversation. US-China Education Review, 3(3), 57-63. 